\title{
Towards Energy-Efficient HealthCare: an Internet-of-Things Architecture Using Intelligent Gateways
}

\author{
Jose Granados ${ }^{1}$, Amir-Mohammad Rahmani ${ }^{1}$, Pekka Nikander ${ }^{2}$, Pasi Liljeberg ${ }^{1}$, and Hannu Tenhunen ${ }^{1}$ \\ ${ }^{1}$ Department of Information Technology, University of Turku, Turku, Finland \\ ${ }^{2}$ Department of Computer Science and Engineering, Aalto University, Helsinki, Finland \\ \{josgra, amirah, pakrli, hatenhu\}@utu.fi, pekka.nikander@aalto.fi
}

\begin{abstract}
The advancement of Internet of Things technologies is enabling continuous monitoring of patients at home and in hospitals. However, Internet of Things architectures still face many challenges regarding power efficiency and scalability at a low cost in order to realize massive ubiquitous deployment, especially in clinical environments. In this paper the benefits of combining data and power into a single cable that the Power over Ethernet standard provides have been leveraged by designing a Power over Ethernet enabled Internet of Things gateway which delivers both cloud connectivity and energy to medical sensors and smart hospital appliances. In addition, the gateway has been customized for processing and transmission of health related data in order to alleviate the processing overhead that wireless sensors have allowing them to extend the battery lifetime and overall system energy efficiency and performance.
\end{abstract}

Keywords- Internet of Things; Power over Ethernet; Healthcare; Smart Hospital; Home care; Smart Gateway

\section{INTRODUCTION}

The Internet of Things (IoT) represents the next logical step in the evolution of information technology after mobile communications by connecting everyday objects to the Internet. This physical and virtual "things" that have identities and personalities are part of a self-configuring network infrastructure capable of ubiquitous computing in order to make everyday tasks easier for users of different industries [1]. With the development of IoT technologies, one of the most important areas covered is healthcare. The IoT-health aims to improve the quality of human life by automating tasks previously performed by humans. In that sense, the IoT enables Electronic Health (eHealth), Mobile Health (mHealth) and Ambient Assisted Living (AAL) that allow remote monitoring and tracking of patients living alone at home or treated in hospitals. Data generated from sensors attached to patients is made available to doctors, family and interested parties giving them the ability to check vital signs from anywhere at any time [2] as well as performing intelligent decision making for assisting health workers.

In healthcare, when patients are admitted to a hospital, their mobility is typically reduced to the facilities of the clinic. Those patients that need active monitoring spend most of their time inside a hospital room. The stationary nature and reliability requirements of such healthcare situations allows leveraging an IoT architecture based on devices that are permanently or semi- permanently fixed at their present locations and wired to the rest of the system. At the same time, such wireline systems may be extended to the wireless domain for monitoring mobile patients through local, low-energy wireless standards such as IEEE 802.15 .4 , as long as the nearby medical equipment are not overly sensitive to the resulting radio noise. In wireline, wireless, and hybrid situations, an IoT gateway could play an important role in health-related IoT applications. Initially, such a gateway may securely and reliably bridge legacy sensors and other medical equipment to traditional Internet Protocol (IP) based networks, such as a hospital intranet. In the longer run, such gateways may connect the devices to a (private) cloud-based service, allowing data aggregation and sensor fusion across multiple sensors and all patients, both in hospitals and home care environments. The resulting big data may in turn help with early determination of, for example, viral outbreaks or spread of antibiotics resistant bacteria. However, such potential computational advances lie beyond the scope of this paper.

In most situations, the power requirements of the proposed IoT gateway would be relatively modest, from a few watts to a few tens of watts. Hence, utilizing the well-established Power over Ethernet (PoE) standard to transfer both data and provide power over a single inexpensive cable results in a cost-effective powering solution. Especially in green field installations, such practice has the potential of saving well over half of the cabling costs, due to the lower cost of the Ethernet cable compared to standard power cables, while at the same time increasing communications reliability and reducing radio noise compared to a situation where the communication takes place over a wireless link. In most cases, the total power needed both by IoT communications and intelligence and the sensors and other medical devices is well below $100 \mathrm{~W}$, the future limit of a single Power-over-Ethernet connection. Hence, the power delivered by the Ethernet cable can be used also as an energy source for powering the bio-feedback sensors.

Another potential benefit of the proposed approach is that sensors and actuators for building automation may also be powered and networked by the same PoE enabled technology. This allows building a Smart Hospital system where the medical devices and building automation, including safety and security, may become integrated.

In this paper, we propose an IoT architecture for healthcare applications based on an energy-efficient and resilient gateway 
design taking advantage of the stationary characteristics of many home and hospital environments.

The proposed architecture focuses on enabling wide-spread data collection from both wireless and wireline sensors, not only from the medical world but also from e.g. the building safety and security systems, in a unified way. This framework allows the implementation of IoT healthcare applications, such as remote patient monitoring and elderly home care, as well as smart hospital appliances and eventually smart hospitals where the building itself is a vital part of the system. The combination of this two realms of healthcare services and smart hospital realizations by taking advantage of $\mathrm{PoE}$ and web of things technologies represent a convenient, energy-efficient and costeffective approach to healthcare services given in static scenarios.

The rest of this paper is structured as follows. In Section II, the related work is reviewed. Section III explains the motivation behind the work, Section IV describes the architecture and its components. Section V provides the experimental setup that verifies the practicality and effectiveness of the proposed approach. Finally, Section VI concludes the document.

\section{RELATED WORK}

The concepts of Wireless Body Area Sensor Networks capable of pervasive continuous monitoring of vital sign data with cloud connectivity and low power optimization has been well discussed [3] [4] [5], however in these works the research effort is focused on interfacing networks of medical sensors to web services as well as optimizing data transmission in the network, yet none consider the use of PoE technologies for energy efficiency and intelligent processing of health related data on gateways. Also they assume general application scenarios rather than benefit from the fixed nature of hospitals and homes. Ultimately the gateways in these works are used as bridges to external web servers instead of embedding the web connectivity and signal processing on the gateway itself as proposed in this paper.

\section{MOTIVATION}

IoT applications are based on the extension of Internet to resource-constrained network nodes that cannot take advantage of existing web services due to their high overhead and intricacy, resulting in short-lived communications only. However in the case of medical sensors, the monitoring bio-signals necessitates a constant connection for the non-stop transmission of live data from continuous and pulsed signals such as Electrocardiography (ECG), Electromyography (EMG), Electroencephalography (EEG), respiratory and heart rate. Each signal has a different bandwidth as shown in Table 1 .

Home health and bedside monitoring include elderly, chronic, wounded and users in rehabilitation. This type of care requires medical measurements that can be done with wearable, nearby or stationary sensors from short to long terms (days to years) [6]. Sensors are also expected to function maintenance free, so power consumption needs to remain low due to limitations on batteries and energy harvesting modules which require the sensors to stay in sleep mode most of the time. Many of these functions could be performed on the gateway in order to alleviate the burden that the sensor can carry, taking advantage of the fact that the gateway has less power and bandwidth constraints due to the PoE connection.

User also pay attention to level of comfort provided by the hospital and the ability to carry on with normal daily routine at home. Key factors include communication means, care, integration of personal smartphones and tablets to the collected health data and clinical history. In addition, patients care about individual room control such as climatic conditions, lighting coming in to the room by opening windows and artificial lighting. Enabling the patient to control these parameters frees hospital personnel of having to take care of it.

TABLE I. Types of bio-signals.

\begin{tabular}{|c|c|c|c|}
\hline $\begin{array}{c}\text { Type of } \\
\text { biosignal }\end{array}$ & $\begin{array}{c}\text { Bandwidth } \\
(\mathrm{Hz}) \\
\end{array}$ & $\begin{array}{c}\begin{array}{c}\text { Quantization } \\
\text { (bits) }\end{array} \\
\end{array}$ & Sensor type \\
\hline ECG & $0.15-100$ & $12-16$ & $\begin{array}{c}\text { Disposable } \\
\mathrm{Ag} / \mathrm{AgCl} \text { electrode }\end{array}$ \\
\hline EEG & $1-50$ & 24 & $\begin{array}{c}\text { Sintered } \mathrm{Ag} / \mathrm{AgCl} \\
\text { electrode }\end{array}$ \\
\hline EMG & $20-500$ & $12-16$ & $\begin{array}{c}\text { Disposable } \\
\mathrm{Ag} / \mathrm{AgCl} \text { electrode }\end{array}$ \\
\hline $\begin{array}{c}\text { Blood } \\
\text { pressure }\end{array}$ & $0-60$ & $12-16$ & $\begin{array}{l}\text { Arm cuff and } \\
\text { pressure sensor }\end{array}$ \\
\hline $\begin{array}{l}\text { Respiration } \\
\text { rate }\end{array}$ & $0-10$ & 10 & $\begin{array}{l}\text { Belt/Nose flow } \\
\text { sensor }\end{array}$ \\
\hline Heart rate & $0-20$ & 10 & Photoelectric \\
\hline $\begin{array}{l}\text { Blood } \\
\text { glucose }\end{array}$ & $\begin{array}{l}\text { Discrete } \\
\text { measure- } \\
\text { ments }\end{array}$ & - & $\begin{array}{c}\text { Disposable } \\
\text { electrode with } \\
\text { glucose oxidase } \\
\text { enzyme }\end{array}$ \\
\hline
\end{tabular}

\section{ENERGY-EFFICIENT IOT ARCHITECTURE FOR HEALTHCARE}

Internet of Things (IoT) gateways link nodes of connected devices to WAN IP networks and web applications, becoming one of the cornerstones of the system. The energy-efficient and intelligent gateway proposed features components customized for the processing and transmission of healthcare related signals and smart hospital appliances.

The system architecture is shown in Fig. 1. The medical sensor nodes used for assessing physiological status of patients serve as one of the data inputs to the system. These are wired or wireless sensors for biometrics such as ECG, EMG, EEG, temperature, pressure, heart rate, position, respiration rate, glucose levels, complete blood count, etc. These sensors are connected to the gateway which executes optional signal processing and/or feature extraction and forwards relevant information to a server, database or a directly to a web client.

In addition to remote patient monitoring, a smart building system is integrated consisting of IoT nodes used for building automation and control systems for hospitals. These include traditional lighting, public address, access control and HVAC as well as hospital centric appliances namely next-to-bed controls and context aware applications such as intelligent nurse calling [10].

The envisioned system is environmentally friendly by harnessing the PoE capabilities of the gateway, which allows for powering sensors and actuators directly without recurring to a different power grid or AC outlets. This in conjunction with the low-cost characteristics enables the massive deploying of 
gateways across the facilities. At first, the system is designed so one gateway is dedicated to one patient, taking care of all sensors/devices attached to it. However, the gateway is sufficiently low-cost to allow a greater degree of distribution which also increases the overall wireless bandwidth for the wireless sensors.

The back-end of the system consists of the remaining components: the PoE enabled switch, a cloud computing platform that includes broadcasting, data warehouse and Big Data analytics servers, and finally Web clients as end users.
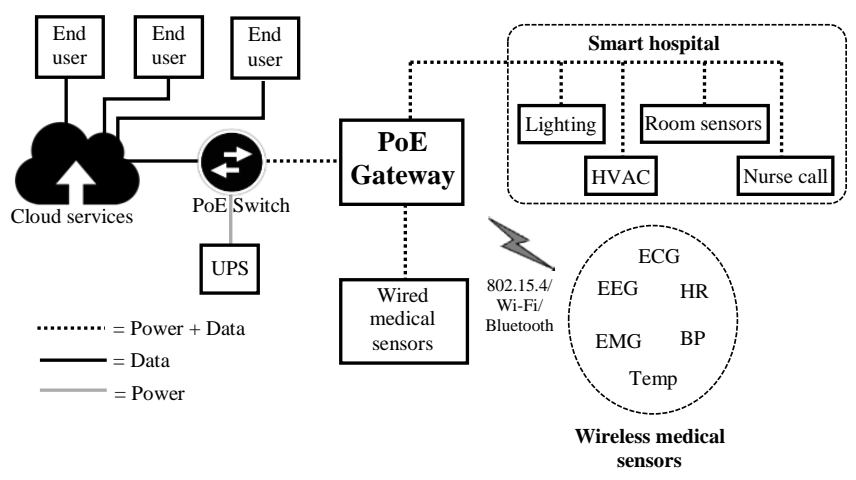

Figure 1. Energy-efficient IoT-health architecture.

\section{A. Intelligent PoE Gateway}

Medical sensors capture signals from the body used for treatment and diagnosing of a medical state. The gateway then receives the captured data from the sensors and performs data aggregation, filtering and dimensionality reduction to avoid clogging the network with noisy or redundant data. Given the stationary nature of clinical and home environments the sensors could be wired directly to the gateway and powered from it.

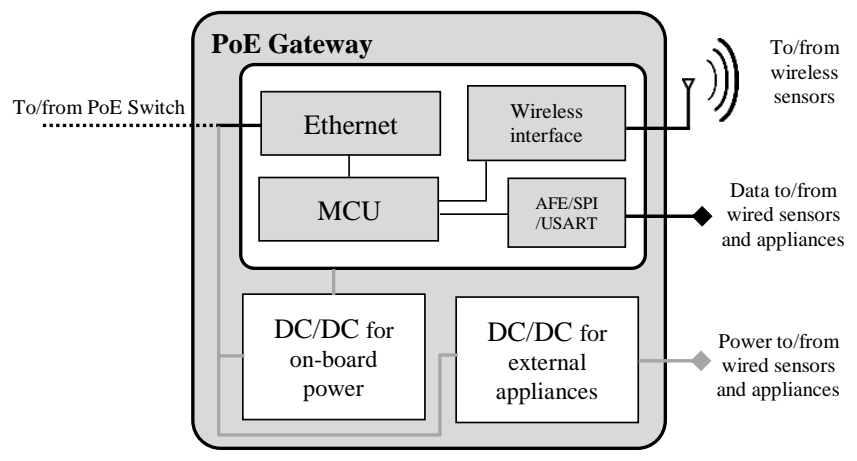

Figure 2. Intelligent PoE gateway for IoT-health.

The gateway receives commands over Internet that are proxied to sensors and actuators. An example command is to change the mode of a sensor from local data logger to web streaming. Commands are then translated to the appropriate sensor application and network layer protocols e.g. CoAP, 6LoWPAN or Bluetooth. Once the sensor processes the command and replies, the response is sent back to the gateway which again proxies the data to the Web client. The gateway has Power over Ethernet so that power and data run through one cable with the benefits of less cluttered installation in stationary settings such as clinics and home.

We have designed a hardware platform known as Ell-i [7] to serve as a PoE gateway. Ell-i is an open source Arduino like development platform for fixed things that implements IEEE 802.3at [8] Power-over-Ethernet standard for 1-100 watts power consumption range. In addition, a DC/DC converter on board converts $48 \mathrm{~V}$ into $5 \mathrm{~V}$ and/or 3.3V needed by the Microcontroller Unit (MCU), and an additional power supply converter is used for providing bulk power to the wired sensors and actuators of the system such as high-power LEDs, solenoids or electric motors. Ell-i is based on a STM32F051R8 ARM Cortex-M0 MCU and provides Internet connectivity via 10BASE-T Ethernet port controlled by an ENC28J60 [9] module connected to the MCU via SPI serial interface. Fig. 2 shows the components of the gateway. Fig. 3 depicts the Ell-i platform with a Bluetooth daughterboard to interface Bluetooth sensors.

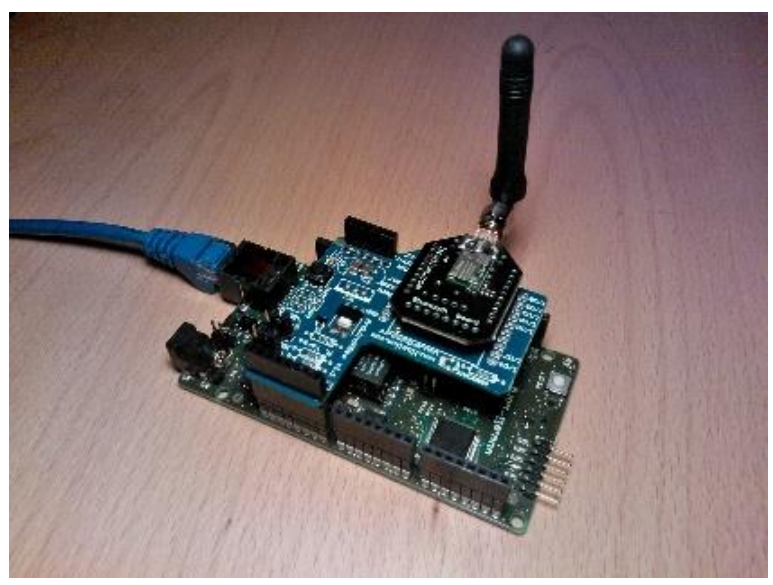

Figure 3. Powe over Ethernet gateway (Ell-i) with Bluetooth daugter board.

The TCP/IP stack was designed to provide minimal functionality for small memory footprint due to the MCU memory constraints and as a way to reduce cost since memory area is the most expensive factor in silicon. The embedded software stack is shown in Fig. 4.

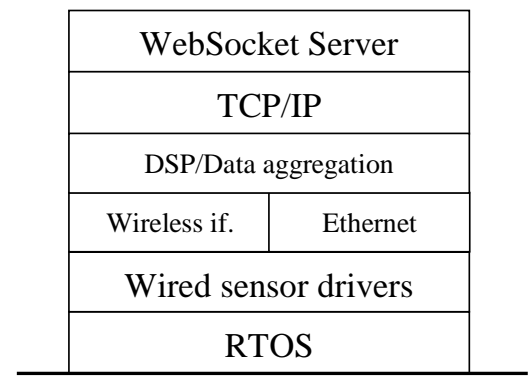

Figure 4. IoT Gateway runtime stack

\section{B. Integration of Smart Hospital appliances}

The networking capabilities of the gateway can serve multiple roles in addition to monitoring patients. The same protocols used for connecting medical sensors are used in a similar way for managing building automation systems such as lighting, access control, indoor PA systems and HVAC controls and other more clinical centric as in the case of ambient- 
intelligent nurse calling systems. This setup enables the centralization of data acquisition not only of patient health data but also of hospital building management for an integrated Smart Hospital framework.

The gateway serves not only as a managing node for the smart hospital controls but also as a smart power source by harnessing the power coming from the PoE cable. This makes the system more energy-efficient and practical compared to other architectures that use embedded solutions such as Raspberry Pi [11] as web servers.

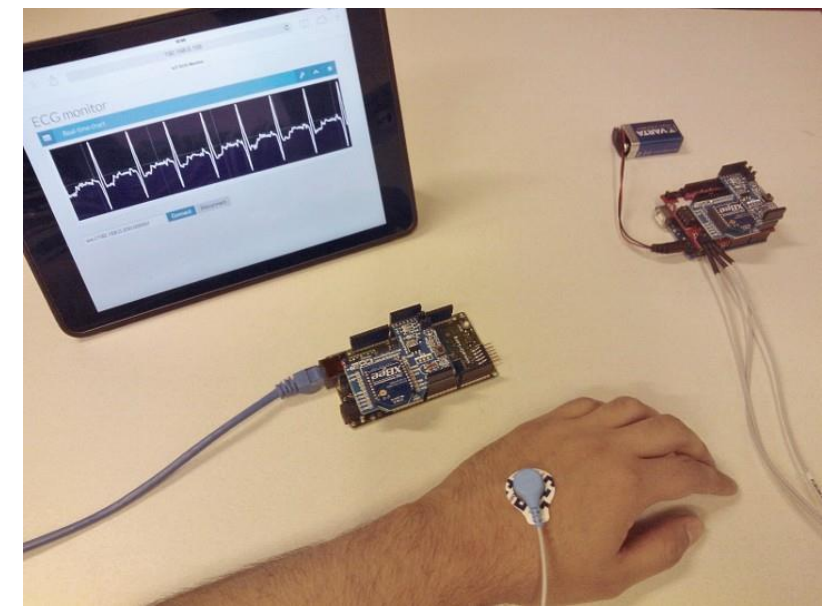

Figure 5. Experimental setup including wireless sensor, PoE gateway and a tablet device as a Web client.

\section{EVALUATION}

We have implemented an experimental setup as shown in Fig. 5 in order to evaluate the monitoring system in terms of sampling rate, latency, cost and convenience. In this test, a medical sensor for ECG signal acquisition has been developed based on an Arduino UNO platform together with eHealth and Xbee daughter boards. An Arduino based-ECG system serves as a platform to acquire signal data to be then transmitted serially. This device emulates most monitoring equipment found in hospitals, with the added benefit of having lower power requirements than traditional medical devices. The wireless sensor includes ECG electrodes with appropriate instrumentation amplifiers and IEEE 802.15.4 wireless transmission capability. The size of the wireless sensor is small enough to make it portable, and could be made smaller with a redesign of the PCB. The battery powered sensor acquires the ECG signal at 500 samples per second using the integrated 10 bit ADC of an ATmega328 microcontroller, and transmits it immediately as it is sampled in two words of 8 bits each. The transmission proves to be reliable for fixed environments such as inside a room given the fact that the test patient can move around $20 \mathrm{~m}$ around where the gateway is located without any noticeable lag in transmission. The gateway receives the sensor ECG signal data through its own 802.15.4 wireless interface and applies a low-pass FIR filter for removing noise and a high-pass IIR filter for removing baseline wandering. Then the processed signal is served from the gateway to cloud services and Web clients through an embedded WebSocket server and Ethernet interface with a custom TCP/IP stack developed for this task. The data is buffered to 400 samples and sent into messages of
800 bytes each averaging a data rate of $1.1 \mathrm{~KB} / \mathrm{s}$. This represents a delay of $800 \mathrm{~ms}$ between the sampling and the transmission to the cloud. In our LAN setup, it takes $32 \mathrm{~ms}$ for the Web client to receive the packet and render the continuous chart resulting in a total latency of around $900 \mathrm{~ms}$. The buffer size can be decreased to lower the latency at the expense of higher processing overhead for the Web Client. The energy source for the gateway is provided by the PoE enabled Ethernet cable.

\section{CONCLUSIONS}

We have developed an IoT architecture for healthcare based on PoE enabled gateways. This benefits fixed or semifixed scenarios where the patients are usually confined to one room and therefore, technologies such as PoE could be leveraged. We have demonstrated the feasibility of the system by developing $E l l-i$, a hardware/software platform serving as a medical wireless sensor gateway and intelligent processing hub that is designed to process health related data and expose it to cloud services and web clients through an embedded WebSocket server. The proposed gateway design performs digital filtering of vital signs signals, as well as streaming to Web clients. The powering capabilities of the gateway can also serve as a power + data source for wired sensors as well as building automation appliances. The gateway is designed to be low-cost and allows massive deployment along hospital facilities. The gateway together with PoE enabled network infrastructure results in an energy-efficient and low-cost architecture for healthcare in hospitals and homes.

\section{REFERENCES}

[1] Singh, D. et al., "A survey of Internet-of-Things: Future vision, architecture, challenges and services," 2014 IEEE World Forum on Internet of Things (WF-IoT), March 2014.

[2] Lopez, P. et al., "Survey of Internet of Things Technologies for Clinical Environments," 2013 27th International Conference on Advanced Information Networking and Applications Workshops (WAINA), March 2013.

[3] Xiaoya Xu, M. Z. "Wireless Body Sensor Networks with Frontier and Future Development of Information Technology in Medicine and Education.” En Q. J. Shaozi Li, Frontier and Future Development of Information Technology in Medicine and Education, 2013.

[4] Wan, J. et al., "Cloud-enabled wireless body area networks for pervasive healthcare," Network, IEEE, September-October 2013.

[5] Zhong-Yi Jin et al., "A gateway node with duty-cycled radio and processing subsystems for wireless sensor networks." ACM Transactions on Design Automation of Electronic Systems, January 2009

[6] Jianchu Yao, Steve Warren, "Applying the ISO/IEEE 11073 Standards to Wearable Home Health Monitoring Systems," Journal of Clinical Monitoring and Computing, December 2005.

[7] Pekka Nikander et al., "Ell-i: An Inexpensive Platform for Fixed Things." SCPE journal, 2013

[8] IEEE 802.3at standard for Ethernet. Amendment 3: Data terminal equipment (DTE) power via the media dependent interface (MDI) enhancements. IEEE Computer Society, September 2009.

[9] ENC28J60 Data Sheet - Stand-Alone Ethernet Controller with SPI Interface. Microchip Technology Inc., West Chandler Blvd., Chandler, Arizona, USA, 2012.

[10] Ongenae, F. et al., "User-driven design of a context-aware application: An ambient-intelligent nurse call system," Pervasive Computing Technologies for Healthcare (PervasiveHealth), 2012 6th International Conference on, May 2012

[11] Richardson, M. Wallace, S.: "Getting Started with Raspberry Pi.” O'Reilly Media, Inc., 2012. 